\title{
Survey on Recent Ulam Stability Results Concerning Derivations
}

\author{
Janusz Brzdęk, ${ }^{1}$ Liviu Cădariu, ${ }^{2}$ Krzysztof Ciepliński, ${ }^{3}$ Ajda Fošner, ${ }^{4}$ and Zbigniew Leśniak ${ }^{1}$ \\ ${ }^{1}$ Department of Mathematics, Pedagogical University, Podchorązych 2, 30-084 Kraków, Poland \\ ${ }^{2}$ Department of Mathematics, Politehnica University of Timişoara, Piaţa Victoriei 2, 300006 Timişoara, Romania \\ ${ }^{3}$ Faculty of Applied Mathematics, AGH University of Science and Technology, Mickiewicza 30, 30-059 Kraków, Poland \\ ${ }^{4}$ Faculty of Management, University of Primorska, Cankarjeva 5, SI-6104 Koper, Slovenia \\ Correspondence should be addressed to Janusz Brzdęk; jbrzdek@up.krakow.pl \\ Received 5 April 2016; Accepted 28 June 2016 \\ Academic Editor: Shanhe Wu \\ Copyright (C) 2016 Janusz Brzdęk et al. This is an open access article distributed under the Creative Commons Attribution License, \\ which permits unrestricted use, distribution, and reproduction in any medium, provided the original work is properly cited.
}

This is a survey presenting the most significant results concerning approximate (generalized) derivations, motivated by the notions of Ulam and Hyers-Ulam stability. Moreover, the hyperstability and superstability issues connected with derivations are discussed. In the section before the last one we highlight some recent outcomes on stability of conditions defining (generalized) Lie derivations.

\section{Introduction}

We say that a functional equation $\mathscr{E}$ is stable if any function $f$, satisfying the equation approximately, is near to an exact solution of $\mathscr{E}$. A classical question in the theory of functional equations is

under what conditions a functional equation $\mathscr{E}$ is stable?

This problem was formulated by Ulam in 1940 for group homomorphisms (see $[1,2])$. One year later, Ulam's problem was affirmatively solved by Hyers [3] for the Cauchy functional equation $f(a+b)=f(a)+f(b)$. This gave rise to the stability theory of functional equations. Aoki [4] and Rassias [5] considered mappings $f$ from a normed space into a Banach space such that the norm of the Cauchy difference is bounded by the expression $\varepsilon\left(\|a\|^{p}+\|b\|^{p}\right)$ for all $a, b$, some $\varepsilon \geqslant 0$, and $0 \leqslant p<1$. That result was later extended also for real $p \notin[0,1]$. For a survey on recent progress in that area, we refer to [6].

The terminology Hyers-Ulam-Rassias stability indeed has been motivated by [5] and its influence on the research in that area of other mathematicians.

In the last few decades, several stability problems of various functional equations have been investigated by many mathematicians. The reader is referred to monographs [7-9] for a comprehensive account of the subject.

\section{Auxiliary Information}

Let $\mathscr{A}$ be a subring of a ring $\mathscr{B}$. An additive mapping $d: \mathscr{A} \rightarrow$ $\mathscr{B}$ is called a derivation if

$$
d(x y)=d(x) y+x d(y), \quad x, y \in \mathscr{A} .
$$

Recall that in the case when $\mathscr{A}$ and $\mathscr{B}$ are algebras over a field $\mathbb{F}$, some authors define derivations as $\mathbb{F}$-linear (i.e., linear over the field $\mathbb{F}$ ) mappings satisfying the above equality.

If $d$ is a derivation and $g: \mathscr{A} \rightarrow \mathscr{B}$ is an additive mapping satisfying

$$
g(x y)=g(x) y+x d(y), \quad x, y \in \mathscr{A},
$$

then $g$ is called a generalized derivation. In the framework of pure algebra, this concept was first introduced by Brešar [10]. In the context of operator algebras, these mappings first appeared in [11].

Generalized derivations are one of the natural generalizations of ordinary derivations. It is easy to see that generalized derivations are exactly those additive mappings $g$ which can be written in the form $g=d+f$, where $d: \mathscr{A} \rightarrow \mathscr{B}$ is a derivation and $f: \mathscr{A} \rightarrow \mathscr{B}$ is a left centralizer, that is, additive mapping with the property

$$
f(a b)=f(a) b, \quad a, b \in \mathscr{A} .
$$

For results concerning (generalized) derivations, we refer the reader to $[12,13]$ and references therein. 
Singer and Wermer [14] obtained a fundamental result which initiated investigations on the ranges of linear derivations on Banach algebras. The so-called Singer-Wermer theorem states that any continuous linear derivation on a commutative Banach algebra maps into the Jacobson radical. They also made a very insightful conjecture that the assumption of continuity is unnecessary. This conjecture, known as the Singer-Wermer conjecture, was proved in 1988 by Thomas [15]. The Singer-Wermer conjecture implies that any linear derivation on a commutative semisimple Banach algebra is identically zero [16]. More than two decades later, Hatori and Wada [17] proved that the zero operator is the only derivation on a commutative semisimple Banach algebra with the maximal ideal space without isolated points. Based on these facts and a private communication with Watanabe [18], Miura et al. [18] proved the Hyers-Ulam-Rassias stability and Bourgin-type superstability of derivations on Banach algebras. Here we have to point out that the first superstability result concerning derivations on algebras of bounded linear operators on a real or complex infinite dimensional Banach space was obtained already in 1994 by Šemrl [19] (see Section 3, where superstability of derivations is defined). A notion somewhat stronger than superstability is that of hyperstability; it also will be discussed in this survey.

\section{Stability of Derivations}

In 1996, the stability of derivations was considered by Jun and Park [20]. Motivated by the study of Johnson [21, 22], who investigated almost multiplicative maps on Banach algebras (see also [23, 24]), Jun and Park proved that there exists a derivation near an almost derivation from a Banach algebra $C^{n}[0,1]$ of differentiable functions to a finite dimensional Banach $C^{n}[0,1]$-module $\mathscr{M}$ (i.e., a Banach space $\mathscr{M}$ together with a continuous homomorphism $\rho: C^{n}[0,1] \rightarrow \mathscr{B}(\mathscr{M})$, where $\mathscr{B}(\mathscr{M})$ denotes the algebra of all bounded operators on $\mathscr{M})$. More precisely, they showed the following theorem [20, Theorem 5].

Theorem 1. Let $\mathscr{M}$ be a Banach $C^{n}[0,1]$-module and let $\varepsilon>0$. If a mapping $D: C^{n}[0,1] \rightarrow \mathscr{M}$ is continuous and linear such that

$$
\begin{array}{r}
\|D(f g)-\rho(f) D(g)-\rho(g) D(f)\| \leqslant \varepsilon\|f\|\|g\|, \\
\qquad, g \in C^{n}[0,1]
\end{array}
$$

and $\rho(z)^{i} D\left(z^{j}\right)=0$, for $i+j=n+1, i, j=0,1, \ldots, n$, then there exist a continuous linear mapping $d: C^{n}[0,1] \rightarrow M$ and $\varepsilon^{\prime}>0$ such that

$$
\begin{gathered}
d(f g)=\rho(f) d(g)+\rho(g) d(f), \\
f, g \in C^{n}[0,1], \\
\|D(f)-d(f)\| \leqslant \varepsilon^{\prime}\|f\|, \quad f \in C^{n}[0,1],
\end{gathered}
$$

where $\varepsilon^{\prime}$ tends to 0 as $\varepsilon$ tends to 0 .

During the past few years, approximate derivations were studied by a number of mathematicians (see [25-31] and references therein). In the year 2004, Park [28] showed that for an almost derivation $D$ on a complex Banach algebra $\mathscr{A}$ there exists a unique derivation $d$ on $\mathscr{A}$ which is near $D$. From Park's result we can derive the next theorem.

Theorem 2. Let $\mathscr{A}$ be a closed subalgebra of a complex Banach algebra $\mathscr{B}$ and let $D: \mathscr{A} \rightarrow \mathscr{B}$ be a mapping with $D(0)=0$ for which there exists a function $\varphi: \mathscr{A}^{4} \rightarrow[0, \infty)$ such that

$$
\begin{aligned}
& \widetilde{\varphi}(x, y, z, w):=\sum_{j=0}^{\infty} 2^{-j} \varphi\left(2^{j} x, 2^{j} y, 2^{j} z, 2^{j} w\right)<\infty \\
& \| D(\lambda x+\lambda y+z w)-\lambda D(x)-\lambda D(y)-D(z) w \\
& \quad-z D(w) \| \leqslant \varphi(x, y, z, w)
\end{aligned}
$$

for all $\lambda \in \mathbb{T}^{1}:=\{\lambda \in \mathbb{C}:|\lambda|=1\}$ and all $x, y, z, w \in \mathscr{A}$. Then there exists a unique $\mathbb{C}$-linear derivation $d: \mathscr{A} \rightarrow \mathscr{B}$ such that

$$
\|D(x)-d(x)\| \leqslant \frac{1}{2} \widetilde{\varphi}(x, x, 0,0), \quad x \in \mathscr{A} .
$$

One can apply this result to $C^{*}$-algebras and $J B^{*}$-algebras as well (see [28]). Analogously, the Hyers-Ulam-Rassias stability of Lie *-derivations on Lie $C^{*}$-algebras was proved in [29].

Two years after Park's paper on the stability of derivations was published, Badora [26] investigated approximate derivations on Banach algebras using Hyers's theorem (see [3] or [7]). We present Badora's main result [26, Theorem 2] in a bit extended form (in [26], $D, d: \mathscr{A} \rightarrow \mathscr{B}$, where $\mathscr{A}$ is a closed subalgebra of a Banach algebra $\mathscr{B})$. A sketch of its proof will be given later in this section.

Theorem 3. Let $\mathscr{A}$ be a Banach algebra and let $M$ be a Banach $\mathscr{A}$-bimodule. Suppose that $D: \mathscr{A} \rightarrow \mathscr{M}$ is a mapping satisfying

$$
\begin{aligned}
& \|D(x+y)-D(x)-D(y)\| \leqslant \varepsilon, \\
& \|D(x y)-D(x) y-x D(y)\| \leqslant v
\end{aligned}
$$

for all $x, y \in \mathscr{A}$ and some constants $\varepsilon, v>0$. Then there exists a unique derivation $d: \mathscr{A} \rightarrow \mathscr{M}$ such that

$$
\|D(x)-d(x)\| \leqslant \varepsilon, \quad x \in \mathscr{A} .
$$

Moreover, we have

$$
y(D(x)-d(x))=(D(x)-d(x)) y=0 \quad x, y \in \mathscr{A} .
$$

A generalization of Hyers's theorem given by Isac and Rassias (see $[7,32]$ ) shows that if a function $\varphi: \mathbb{R}_{+} \rightarrow \mathbb{R}_{+}$ (here, $\mathbb{R}_{+}$denotes the set of all nonnegative real numbers) satisfies
(i) $\lim _{t \rightarrow \infty}(\varphi(t) / t)=0$,
(ii) $\varphi(t s) \leqslant \varphi(t) \varphi(s), t, s>0$,
(iii) $\varphi(t)<t, t>1$ 
and if $D: \mathscr{A} \rightarrow \mathscr{M}$ is a mapping such that

$$
\begin{array}{r}
\|D(x+y)-D(x)-D(y)\| \leqslant \varepsilon(\varphi(\|x\|)+\varphi(\|y\|)), \\
x, y \in \mathscr{A},
\end{array}
$$

for some positive constant $\varepsilon$, then there exist a unique additive mapping $d: \mathscr{A} \rightarrow \mathscr{M}$ and a constant $c \in \mathbb{R}$ such that

$$
\|D(x)-d(x)\| \leqslant \operatorname{c\varepsilon } \varphi(\|x\|), \quad x \in \mathscr{A} .
$$

The classical example of a function $\varphi$ fulfilling the above three conditions is a map $\varphi(t)=t^{q}, t \in \mathbb{R}_{+}$, where $q<1$. Using this result, we have the following generalization of Theorem 3 .

Theorem 4. Let $\mathscr{A}$ be a Banach algebra and let $M$ be a Banach $\mathscr{A}$-bimodule. Assume that $\varphi_{1}: \mathbb{R}_{+} \rightarrow \mathbb{R}_{+}$is a function with properties ( $i$ ), (ii), and (iii) and $\varphi_{2}: \mathbb{R}_{+} \rightarrow \mathbb{R}_{+}$fulfills $(i)$. Suppose that $D: \mathscr{A} \rightarrow \mathscr{M}$ is a mapping satisfying

$$
\begin{aligned}
& \|D(x+y)-D(x)-D(y)\| \\
& \quad \leqslant \varepsilon\left(\varphi_{1}(\|x\|)+\varphi_{1}(\|y\|)\right), \\
& \|D(x y)-D(x) y-x D(y)\| \leqslant \varphi_{2}(\|x\| \cdot\|y\|)
\end{aligned}
$$

for all $x, y \in \mathscr{A}$ and some constant $\varepsilon>0$. Then there exist $a$ unique derivation $d: \mathscr{A} \rightarrow \mathscr{M}$ and a constant $c \in \mathbb{R}$ such that

$$
\|D(x)-d(x)\| \leqslant c \varepsilon \varphi_{1}(\|x\|), \quad x \in \mathscr{A}
$$

If we want to extend Theorem 4 to the case of $p, q>1$, then we can adopt the method presented by Gajda [33] to obtain the above Isac-Rassias result for the function $\varphi: \mathbb{R}_{+} \rightarrow$ $\mathbb{R}_{+}$fulfilling

$$
\begin{aligned}
& \left(\mathrm{i}^{\prime}\right) \lim _{t \rightarrow 0}(\varphi(t) / t)=0, \\
& \left(\mathrm{ii}^{\prime}\right) \varphi(t s) \leqslant \varphi(t) \varphi(s), t, s>0, \\
& \left(\mathrm{iii}^{\prime}\right) \varphi(t)<t, 0<t<1 .
\end{aligned}
$$

After this modification we get the following version of Theorem 4.

Theorem 5. Let $\mathscr{A}$ be a Banach algebra and let $M$ be a Banach $\mathscr{A}$-bimodule. Assume that $\varphi_{1}: \mathbb{R}_{+} \rightarrow \mathbb{R}_{+}$is a function with properties $\left(i^{\prime}\right),\left(i i^{\prime}\right)$, and $\left(i i^{\prime}\right)$ and $\varphi_{2}: \mathbb{R}_{+} \rightarrow \mathbb{R}_{+}$fulfills $\left(i^{\prime}\right)$. Suppose that $D: \mathscr{A} \rightarrow \mathscr{M}$ is a mapping satisfying (13) for all $x, y \in \mathscr{A}$ and some constant $\varepsilon>0$. Then there exist a unique derivation $d: \mathscr{A} \rightarrow \mathscr{M}$ and a constant $c \in \mathbb{R}$ satisfying (14).

In the same year as Badora's result was presented, Moslehian [34] published a paper which is devoted to the study of the Hyers-Ulam-Rassias stability of generalized derivations. In the proof of the result presented below [34, Theorem 2.1], to construct a generalized derivation Moslehian applied the direct method, which was first devised by Hyers [3] constructing an additive function from an approximate one. Moreover, the derivation required in the definition of a generalized derivation is also constructed in this way. Let us also point out that the results in [34] are a generalization of those of Park's papers [28, 29].
Theorem 6. Let $\mathscr{A}$ be a complex normed algebra with a unit 1 and let $\mathscr{M}$ be a Banach $\mathscr{A}$-bimodule such that $1 \cdot x=x \cdot 1=x$ for all $x \in \mathscr{M}$. Suppose that $G: \mathscr{A} \rightarrow \mathscr{M}$ is a mapping with $G(0)=0$ for which there exist a map $D: \mathscr{A} \rightarrow \mathscr{M}$ and $a$ function $\varphi: \mathscr{A}^{4} \rightarrow[0, \infty)$ such that

$$
\begin{aligned}
& \widetilde{\varphi}(x, y, z, w):=\frac{1}{2} \sum_{j=0}^{\infty} 2^{-j} \varphi\left(2^{j} x, 2^{j} y, 2^{j} z, 2^{j} w\right)<\infty \\
& \| G(\lambda x+\lambda y+z w)-\lambda G(x)-\lambda G(y)-G(z) w \\
& -z D(w) \| \leqslant \varphi(x, y, z, w)
\end{aligned}
$$

for all $\lambda \in \mathbb{T}^{1}$ and all $x, y, z, w \in \mathscr{A}$. Then there exists a unique $\mathbb{C}$-linear generalized derivation $g: \mathscr{A} \rightarrow \mathscr{M}$ such that

$$
\|G(x)-g(x)\| \leqslant \frac{1}{2} \widetilde{\varphi}(x, x, 0,0), \quad x \in \mathscr{A} .
$$

In the year 2010, Gordji and Moslehian [27] utilized the notion of module extension to reduce the problem of stability of derivations to the problem of stability of homomorphisms (i.e., additive multiplicative mappings) studied by Badora [35]. In the following we give some more details.

Let $\mathscr{A}$ be a Banach algebra and let $\mathscr{M}$ be a Banach $\mathscr{A}$ bimodule. Then, it is easy to see that $\mathscr{M} \oplus_{1} \mathscr{A}$ is a Banach algebra equipped with the $l_{1}$-norm

$$
\|(x, a)\|=\|x\|+\|a\|, \quad x \in \mathscr{M}, a \in \mathscr{A}
$$

and the product

$$
(x, a)(y, b)=(x \cdot b+a \cdot y, a b),
$$

$$
x, y \in \mathscr{M}, a, b \in \mathscr{A} .
$$

Here, the symbol $\|\cdot\|$ represents the norm on a normed algebra $\mathscr{A}$ and the norm on a normed $\mathscr{A}$-bimodule $\mathscr{M}$, and $\cdot$ denotes the module multiplication on $\mathscr{M}$. The algebra $\mathscr{M} \oplus_{1} \mathscr{A}$ is called a module extension Banach algebra. Furthermore, the projection map $\pi_{1}: \mathscr{M} \oplus_{1} \mathscr{A} \rightarrow \mathscr{M}$ is defined by $(x, a) \mapsto x$. Similarly, the projection map $\pi_{2}: \mathscr{M} \oplus_{1} \mathscr{A} \rightarrow \mathscr{A}$ is defined by $(x, a) \mapsto a$. For more information about Banach modules and module extensions, we refer the reader to $[36,37]$.

Badora's result [35, Theorem 1] implies that if $H: \mathscr{A} \rightarrow$ $\mathscr{M}$ is a mapping satisfying

$$
\begin{array}{r}
\|H(x+y)-H(x)-H(y)\| \leqslant \varepsilon, \\
\|H(x y)-H(x) H(y)\| \leqslant v
\end{array}
$$

for all $x, y \in \mathscr{A}$ and some constants $\varepsilon, v>0$, then there exists a unique homomorphism $h: \mathscr{A} \rightarrow \mathscr{M}$ such that

$$
\|H(x)-h(x)\| \leqslant \varepsilon
$$

for all $x \in \mathscr{A}$. Furthermore,

$$
y(H(x)-h(x))=(H(x)-h(x)) y=0
$$

for all $x \in \mathscr{A}$ and all $y$ in the algebra generated by $h(\mathscr{A})$. 
Now, suppose that $D: \mathscr{A} \rightarrow \mathscr{M}$ satisfies the assumptions of Theorem 3 and let $H_{D}: \mathscr{A} \rightarrow \mathscr{M} \oplus_{1} \mathscr{A}$ be a mapping defined by

$$
H_{D}(x)=(D(x), x), \quad x \in \mathscr{A} \text {. }
$$

Then, it is easy to see that

$$
\left\|H_{D}(x+y)-H_{D}(x)-H_{D}(y)\right\| \leqslant \varepsilon
$$

and similarly

$$
\left\|H_{D}(x y)-H_{D}(x) H_{D}(y)\right\| \leqslant v
$$

for all $x, y \in \mathscr{A}$. Thus, it follows that there exists a unique homomorphism $h: \mathscr{A} \rightarrow \mathscr{M} \oplus_{1} \mathscr{A}$ such that

$$
\left\|H_{D}(x)-h(x)\right\| \leqslant \varepsilon
$$

for all $x \in \mathscr{A}$. Furthermore,

$$
(y, z)(D(x)-d(x))=(D(x)-d(x))(y, z)=0
$$

for all $x \in \mathscr{A}$ and all $(y, z)$ in the algebra generated by $h(\mathscr{A})$. Now, if we put $d:=\pi_{1} \circ h$, then we can show that $d$ satisfies all the requirements in Theorem 3. Similarly, Fošner and Moslehian [38] reduced the problem of stability of generalized derivations to the stability of homomorphisms using the module extension as a trick.

\section{Hyperstability and Superstability of Derivations}

The investigation of the multiplicative and exponential Cauchy functional equations (see [39]; for recent extensions we refer to [40]) highlighted a new phenomenon, which is nowadays called superstability (see, e.g., [41]). In this case the so-called stability inequality implies that the observed function is either bounded or it is a solution of the functional equation. But it can also happen that each function $f$, satisfying the functional equation $\mathscr{E}$ approximately, must actually be a solution of the proposed equation $\mathscr{E}$. In this case we say that the functional equation $\mathscr{E}$ is hyperstable. According to our best knowledge, the first hyperstability result was published in [42] and concerned with homomorphisms. However, the term hyperstability has been used for the first time probably in [43] (see also $[44,45]$ ). For more information about the new results on hyperstability and superstability, we refer the reader to review articles $[46,47]$.

As we are aware, the hyperstability result concerning derivations was first obtained by Šemrl in [19] (actually, it has been named there a superstability result).

Theorem 7. Let $\mathscr{B}(X)$ be the algebra of all bounded linear operators on a real or complex infinite dimensional Banach space $X$ and let $\mathscr{A}(X)$ be a standard operator algebra on $X$. Assume that $\varphi: \mathbb{R}_{+} \rightarrow \mathbb{R}_{+}$is a function with the property

$$
\lim _{t \rightarrow \infty} \frac{\varphi(t)}{t}=0
$$

Suppose that $D: \mathscr{A}(X) \rightarrow \mathscr{B}(X)$ is a mapping satisfying

$$
\|D(A B)-D(A) B-A D(B)\| \leqslant \varphi(\|A\|\|B\|),
$$

$$
A, B \in \mathscr{A}(X) \text {. }
$$

Then, $D$ is a linear derivation. More precisely, there exists $T \in$ $\mathscr{B}(X)$ such that

$$
D(A)=A T-T A
$$

for all $A \in \mathscr{A}(X)$ (i.e., $D$ is an inner derivation).

Generally the above hyperstability result is not true. Namely, let $\mathscr{M}_{2}$ be the algebra of all $2 \times 2$ real matrices and

$$
\mathscr{A}=\left\{\left[\begin{array}{ll}
x & 0 \\
0 & 0
\end{array}\right]: x \in \mathbb{R}\right\} .
$$

Suppose that $f: \mathscr{A} \rightarrow \mathscr{M}_{2}$ is a mapping given by the formula

$$
f\left(\left[\begin{array}{ll}
x & 0 \\
0 & 0
\end{array}\right]\right)=\left[\begin{array}{ll}
0 & 0 \\
0 & 1
\end{array}\right], \quad x \in \mathbb{R} .
$$

Then,

$$
\begin{gathered}
f\left(\left[\begin{array}{ll}
x & 0 \\
0 & 0
\end{array}\right]+\left[\begin{array}{ll}
y & 0 \\
0 & 0
\end{array}\right]\right)-f\left(\left[\begin{array}{ll}
x & 0 \\
0 & 0
\end{array}\right]\right) \\
-f\left(\left[\begin{array}{ll}
y & 0 \\
0 & 0
\end{array}\right]\right)=\left[\begin{array}{cc}
0 & 0 \\
0 & -1
\end{array}\right], \\
f\left(\left[\begin{array}{ll}
x & 0 \\
0 & 0
\end{array}\right] \cdot\left[\begin{array}{ll}
y & 0 \\
0 & 0
\end{array}\right]\right)-f\left(\left[\begin{array}{ll}
x & 0 \\
0 & 0
\end{array}\right]\right) \cdot\left[\begin{array}{ll}
y & 0 \\
0 & 0
\end{array}\right] \\
-\left[\begin{array}{ll}
x & 0 \\
0 & 0
\end{array}\right] \cdot f\left(\left[\begin{array}{ll}
y & 0 \\
0 & 0
\end{array}\right]\right)=\left[\begin{array}{ll}
0 & 0 \\
0 & 1
\end{array}\right]
\end{gathered}
$$

for all $x, y \in \mathbb{R}$. Therefore, $f$ satisfies assumptions of Theorem 7 with a constant function $\varphi$ but $f$ is not a derivation (see also [26]). But, since $f$ is bounded, this does not exclude the superstability phenomenon in this case.

In [26, Corollary 1, Theorem 5], Badora studied superstability of additive derivations on unital Banach algebras. The following theorem is a generalization of Badora's result.

Theorem 8. Let $\mathscr{A}$ be a Banach algebra with a unit 1 and let $\mathscr{M}$ be a Banach $\mathscr{A}$-bimodule such that $1 \cdot x=x \cdot 1=x$ for all $x \in \mathscr{M}$. Suppose that $D: \mathscr{A} \rightarrow \mathscr{M}$ is a mapping satisfying

$$
\begin{aligned}
& \|D(x+y)-D(x)-D(y)\| \leqslant \varepsilon, \\
& \|D(x y)-D(x) y-x D(y)\| \leqslant v
\end{aligned}
$$

for all $x, y \in \mathscr{A}$ and some constants $\varepsilon, v>0$. Then $D$ is a derivation.

As in the previous section of our paper, we can generalize Theorem 8 replacing constants $\varepsilon$ and $\nu$ by functions fulfilling corresponding conditions. 
Next, Moslehian [34] proved the hyperstability result for generalized derivations. More precisely, Moslehian studied approximate generalized derivations on unital Banach algebras in the following way [34, Theorem 2.7].

Theorem 9. Let $\mathscr{A}$ be a complex Banach algebra with a unit 1. Suppose that $G: \mathscr{A} \rightarrow \mathscr{A}$ is a mapping with $G(0)=0$ for which there exist a map $D: \mathscr{A} \rightarrow \mathscr{A}$ and a constant $\varepsilon>0$ such that

$$
\begin{aligned}
& \| G(\lambda x+\lambda y+z w)-\lambda G(x)-\lambda G(y)-G(z) w \\
& \quad-z D(w) \| \leqslant \varepsilon
\end{aligned}
$$

for all $\lambda \in \mathbb{T}^{1}$ and all $x, y, z, w \in \mathscr{A}$. Then, $G$ is a $\mathbb{C}$-linear generalized derivation and $D$ is a $\mathbb{C}$-linear derivation.

Later, Gordji and Moslehian [27, Corollary 2, Corollary 3] presented two superstability results concerning derivations as direct consequences of the main result of the paper [27] (i.e., Theorem 3). Similarly, Fošner and Moslehian [38, Corollary 4.5] proved a hyperstability result concerning generalized derivations.

Theorem 10. Let $\mathscr{A}$ be a Banach algebra and let $M$ be a Banach $\mathscr{A}$-bimodule without order. Suppose that $G, D: \mathscr{A} \rightarrow$ $M$ are mappings satisfying

$$
\begin{gathered}
\|D(x+y)-D(x)-D(y)\| \leqslant \varepsilon_{1}, \\
\|G(x+y)-G(x)-G(y)\| \leqslant \varepsilon_{2}, \\
\|D(x y)-D(x) y-x D(y)\| \leqslant v_{1}, \\
\|G(x y)-G(x) y-x D(y)\| \leqslant v_{2}
\end{gathered}
$$

for all $x, y \in \mathscr{A}$ and some constants $\varepsilon_{1}, \varepsilon_{2}, v_{1}, v_{2}>0$. Then, $G$ is a generalized derivation and $D$ is a derivation.

So, if $\mathscr{A}$ is a Banach algebra with an approximate unit and $G, D: \mathscr{A} \rightarrow \mathscr{A}$ mappings satisfying the above conditions for all $x, y \in \mathscr{A}$ and some constants $\varepsilon_{1}, \varepsilon_{2}, \nu_{1}, v_{2}>0$, then $G$ is a generalized derivation and $D$ is a derivation. Namely, every Banach algebra with an approximate unit, as a Banach bimodule over itself, is without order.

The superstability and hyperstability properties of mappings in various classes, related to derivations and generalized derivations, were widely studied by several mathematicians. An interested reader can find more information about new results and further references on the topic in [48-55].

We end this section with an example of hyperstability result, which is a simple characterization of derivations, proved in a recent paper by A. Bahyrycz, J. Brzdęk, and M. Piszczek "Approximately $p$-Wright Affine functions, Inner Product Spaces, and Derivations" to appear in Fixed Point Theory. To this end we need to introduce the following three hypotheses.

$\left(\mathscr{H}_{1}\right)$ For each $x \in \mathscr{A}$ there is a set $D_{x} \subset \mathbb{F}$ such that int $\left(D_{x}-\right.$ $\left.D_{x}\right) \neq \emptyset$ and $g$ is bounded on the set $D_{x} x:=\{a x: a \in$ $\left.D_{x}\right\}$.
$\left(\mathscr{H}_{2}\right)$ There exists $(u, v) \in \mathbb{R}^{2} \backslash([1, \infty) \times[0, \infty))$ such that

$$
\sup _{x \in \mathscr{A} \backslash\{0\}, \alpha \in \mathbb{F}\{\{0\}} \frac{\|g(\alpha x)-\alpha g(x)\|}{|\alpha|^{u}+\|x\|^{v}}<\infty .
$$

$\left(\mathscr{H}_{3}\right)$ There exists $(u, v) \in \mathbb{R}^{2} \backslash\{(1,1)\}$ such that

$$
\sup _{x \in \mathscr{A} \backslash\{0\}, \alpha \in \mathbb{F}\{\{0\}} \frac{\|g(\alpha x)-\alpha g(x)\|}{|\alpha|^{u}\|x\|^{v}}<\infty .
$$

For instance, if a set $D \subset \mathbb{F}$ (with $\mathbb{F} \in\{\mathbb{R}, \mathbb{C}\}$ ) has a positive inner Lebesgue measure or contains a subset of the second category and with the Baire property, then $\operatorname{int}(D-D) \neq \emptyset$ (see, e.g., [56]). For related results we refer to [56-58].

Now we can present the above-mentioned result, corresponding to several outcomes in, for example, $[20,52,59,60]$.

Proposition 11. Let $\mathbb{F} \in\{\mathbb{R}, \mathbb{C}\}$, let $\mathscr{A}$ be a normed algebra over $\mathbb{F}$, let $\mathscr{M}$ be a normed $\mathscr{A}$-bimodule, $\mathscr{A}$ has an element $e$ that is not a zero divisor, and $g: \mathscr{A} \rightarrow \mathscr{M}$. Assume that there exist $p \in \mathbb{F} \backslash\{0,1,1 / 2\}$ and $k, l \in(-\infty, 0)$ such that

$$
\begin{array}{r}
\sup _{x, y \in \mathscr{A} \backslash\{0\}} \frac{\|G(x, y)\|}{\|x\|^{k}+\|y\|^{k}}<\infty, \\
\sup _{x, y \in \mathscr{A} \backslash\{0\}} \frac{\|g(x y)-g(x) y-x g(y)\|}{\|x\|^{l}\|y\|^{l}}<\infty,
\end{array}
$$

where $G(x, y)=g(p x+(1-p) y)+g((1-p) x+p y)-g(x)-$ $g(y)$. Then, $g$ is a derivation. Moreover, if one of hypotheses $\left(\mathscr{H}_{1}\right)-\left(\mathscr{H}_{3}\right)$ is valid, then $g$ is $\mathbb{F}$-linear.

Note that, in the last proposition, the inequality related to the following functional equation of $p$-Wright affine functions

$$
\begin{aligned}
& g(p x+(1-p) y)+g((1-p) x+p y) \\
& \quad=g(x)+g(y)
\end{aligned}
$$

has been used instead of the conditions connected with some kind of stability of the Cauchy equation

$$
g(x+y)=g(x)+g(y) .
$$

\section{Lie Derivations}

In what follows we assume all the time that $\mathscr{A}$ is a normed algebra over the field $\mathbb{F} \in\{\mathbb{R}, \mathbb{C}\}$ and $\mathscr{M}$ is a normed $\mathscr{A}$ bimodule. For all $x \in \mathscr{A}$ and $u \in \mathscr{M}$, the symbols $[x, u]$ and $[u, x]$ denote in $\mathscr{M}$ the commutators $x u-u x$ and $u x-x u$, respectively. Moreover, we say that an additive mapping $d$ : $\mathscr{A} \rightarrow \mathscr{M}$ is a Lie derivation provided

$$
d([x, y])=[d(x), y]+[x, d(y)], \quad x, y \in \mathscr{A} .
$$

The next result has been obtained in the already mentioned paper by Bahyrycz, Brzdęk, and Piszczek (to appear in Fixed Point Theory). 
Proposition 12. Assume that $g: \mathscr{A} \rightarrow \mathscr{M}$ and there exist $p \in \mathbb{Q} \backslash\{0,1,1 / 2\}, k \in(-\infty, 0)$, and $l \in \mathbb{R} \backslash\{1\}$ such that (38) holds and

$$
\sup _{x, y \in \mathscr{A} \backslash\{0\}} \frac{\|g([x, y])-[g(x), y]-[x, g(y)]\|}{\|x\|^{l}\|y\|^{l}}<\infty .
$$

Then, $g$ is a Lie derivation. Moreover, if one of hypotheses $\left(\mathscr{H}_{1}\right)-\left(\mathscr{H}_{3}\right)$ is valid, then $g$ is linear.

In the remaining part of this section we present some outcomes (the last three corollaries) concerning stability of the generalized Lie derivation from [61], where some useful tools have been provided allowing a systematic study of such issues. To this end we must make some preparations.

First, let us recall that, analogously as in the case of derivations, an additive mapping $g: \mathscr{A} \rightarrow \mathscr{M}$ is called a generalized Lie derivation if there exists an additive mapping $d: \mathscr{A} \rightarrow \mathscr{M}$ such that

$$
\begin{array}{r}
g([x, y])=g(x) y-y d(x)+x d(y)-g(y) x, \\
x, y \in \mathscr{A} .
\end{array}
$$

For the sake of precision, given an additive $d: \mathscr{A} \rightarrow \mathscr{M}$, every additive $g: \mathscr{A} \rightarrow \mathscr{M}$ satisfying (44) will be named a generalized Lie $d$-derivation.

Next, let $\mathfrak{B}_{\mathbb{F}}$ denote the family of all sets $\Gamma \subseteq \mathbb{F}$ such that each additive function $f: \mathbb{F} \rightarrow \mathscr{M}$ that is bounded on $\Gamma$ must be continuous. Clearly, if $\Gamma \subset \mathbb{F}$ has a nonempty interior, then $\Gamma \in \mathfrak{B}_{\mathbb{F}}$. This also is true when $\Gamma \subseteq \mathbb{F}$ is of a positive inner Lebesgue measure or has a subset of the second category and with the Baire property (cf. [56]). For more information on $\mathfrak{B}_{\mathbb{F}}$ and further references concerning the subject, we refer the reader to, for example, [56-58].

Let $A_{i} \subset \mathscr{A}, \eta_{i}, \varepsilon_{i} \in[0, \infty)$, and $p_{i} \in \mathbb{R}$ for $i=1,2,3$. Assume yet that $p_{i} \geqslant 0$ or $0 \notin A_{i}$ for $i=1,2,3$. We present a very simple case when $\mathscr{A}$ is a normed Lie algebra and

$$
\begin{aligned}
\varphi_{i}(x, y):=\eta_{i}+\varepsilon_{i}\left(\|x\|^{p_{i}}+\|y\|^{p_{i}}\right) & \\
& x, y \in A_{i}, i=1,2,3 .
\end{aligned}
$$

In what follows, it is assumed all the time that $\Gamma \subset \mathbb{F} \backslash\{0\}$, $\emptyset \neq A \subset \mathscr{A}$, and $\varphi_{1}, \varphi_{2}, \varphi_{3}: \widetilde{A}^{2} \rightarrow[0, \infty)$ are defined by (45), and $g: \widetilde{A}_{0} \rightarrow \mathscr{M}$ and $d: A \rightarrow \mathscr{M}$ are mappings satisfying the conditions

$$
\begin{aligned}
& \|d(\gamma x+y)-\gamma d(x)-d(y)\| \leqslant \varphi_{1}(x, y), \\
& x, y \in A, \gamma \in \Gamma, \gamma x+y \in A, \\
& \|g(\gamma x+y)-\gamma g(x)-g(y)\| \leqslant \varphi_{2}(x, y), \\
& x, y \in \widetilde{A}, \gamma \in \Gamma, \gamma x+y \in \widetilde{A}, \\
& \|g([x, y])-g(x) y+y d(x)-x d(y)+g(y) x\| \\
& \leqslant \varphi_{3}(x, y), \quad x, y \in A .
\end{aligned}
$$

Let us yet recall that $\mathscr{I} \subset 2^{\mathscr{A}}$ is an ideal provided $2^{D} \subset \mathscr{I}$ and $C \cup D \in \mathscr{I}$ for every $C, D \in \mathscr{I}$. Next, given $A \subset \mathscr{A}$, we write $\widetilde{A}_{0}:=A \cup\{[x, y]: x, y \in A\}$ and $\widetilde{A}:=\widetilde{A}_{0} \backslash\{0\}$.
The theorems read as follows.

Theorem 13. Let $\mathscr{I} \subset 2^{\mathscr{A}}$ be an ideal such that

$$
D+x \in \mathscr{I}, \quad D \in \mathscr{I}, x \in \mathscr{A} \text {. }
$$

Let $p_{3} \neq 2, \eta_{3}\left(p_{3}-2\right) \leqslant 0$, and suppose that one of the following three conditions is valid:

(a) $A=\mathscr{A}$, $\Gamma$ is unbounded, $p_{1}>1, p_{2}>1$, and $\eta_{1}=\eta_{2}=$ 0

(b) $A=\mathscr{A} \backslash\{0\}$, $\Gamma$ is unbounded, and $p_{i}<1$ for $i=1,2$;

(c) $p_{i}<0$ for $i=1,2$ and there exists $B \in \mathscr{I}$ with $2 B=B$ and $A=\mathscr{A} \backslash B$.

Then there exist a unique additive mapping $D: \mathscr{A} \rightarrow M$ and a unique generalized Lie D-derivation $G: \mathscr{A} \rightarrow \mathscr{M}$ such that

$$
\begin{aligned}
& G(\gamma x)=\gamma G(x), \\
& D(\gamma x)=\gamma D(x), \\
& x \in \mathscr{A}, \gamma \in \Gamma, \\
& d(x)=D(x), \\
& g(y)=G(y),
\end{aligned}
$$

$$
x \in A, y \in \widetilde{A} .
$$

If $\Gamma$ has a bounded subset from $\mathfrak{B}_{\mathbb{F}}$, then $G$ and $D$ are $\mathbb{F}$-linear.

Theorem 14. Let $A=\mathscr{A}$ and $s:=\sup \{|\gamma|: \gamma \in \Gamma\}<\infty$. If there is $\gamma_{0} \in \Gamma$ with $\left|\gamma_{0}\right|=1, \eta_{1}=\eta_{2}=\eta_{3}=0, p_{1}>1$, $p_{2}>1$, and $p_{3}>2$, then there exist a unique additive mapping $D: \mathscr{A} \rightarrow \mathscr{M}$ and a unique generalized Lie D-derivation $G$ : $\mathscr{A} \rightarrow \mathscr{M}$ such that (48) holds and

$$
\begin{gathered}
\|d(x)-D(x)\| \leqslant \frac{\rho_{1} \varepsilon_{1}\|x\|^{p_{1}}}{1-\rho_{1}}, \\
\|g(x)-G(x)\| \leqslant \frac{\rho_{2} \varepsilon_{2}\|x\|^{p_{2}}}{1-\rho_{2}}
\end{gathered}
$$

for all $x \in \mathscr{A}$, where

$$
\rho_{i}:=\inf \left\{|c|^{p_{i}} s^{1-p_{i}}+|1-c|^{p_{i}}: c \in \mathbb{F}\right\}, \quad i=1,2 .
$$

Moreover, if $\Gamma$ has a subset from $\mathfrak{B}_{\mathbb{F}}$, then $G$ and $D$ are $\mathbb{F}$-linear.

Theorem 15. Let $A=\mathscr{A} \backslash\{0\}, \sigma:=\sup \{|\mu+1|: \mu \in \Gamma\}>1$, $p_{1}<1, p_{2}<1, p_{3}<2$, and suppose that $\Gamma$ is bounded. Then, there are a unique additive mapping $D: \mathscr{A} \rightarrow \mathscr{M}$ and a unique generalized Lie D-derivation $G: \mathscr{A} \rightarrow \mathscr{M}$ such that (48) holds and

$$
\begin{array}{ll}
\|d(x)-D(x)\| \leqslant \frac{2 \varepsilon_{1}\|x\|^{p_{1}}}{\sigma-\sigma^{p_{1}}}+\frac{\eta_{1}}{\sigma-1}, & x \in \mathscr{A} \backslash\{0\}, \\
\|g(x)-G(x)\| \leqslant \frac{2 \varepsilon_{2}\|x\|^{p_{2}}}{\sigma-\sigma^{p_{2}}}+\frac{\eta_{2}}{\sigma-1}, \quad x \in \mathscr{A} \backslash\{0\} .
\end{array}
$$

Moreover, if $\Gamma$ has a subset from $\mathfrak{B}_{\mathbb{F}}$, then $G$ and $D$ are $\mathbb{F}$-linear.

Note that the last two theorems concern some cases that are complementary to those described in (a)-(c). 


\section{Final Remarks}

Besides generalized derivations, there are several other generalizations of the notion of derivations. It seems that they first appeared in the framework of pure algebra (see [62]). Recently they have been treated in the Banach algebra theory as well (see [63]). The stability of those derivations was extensively studied by several authors in, for example, [50, 51, $53,55,64-67]$. We also refer the readers to two papers of C. G. Park (Nonlinear Funct. Anal. Appl. 10 (2005), 751-776 and J. Lie Theory 15 (2005), 393-414) and a paper of C. G. Park and D. Y. Shin (Korean J. Math. 22 (2014), 139-150), which had to be removed from the references in this paper due to some restrictions of the journal.

For example, a discussion of stability of the so-called $(\delta-$ $\tau)$-derivations and a study of the so-called generalized $(\theta, \phi)$ derivations are given in [67] and the above-mentioned paper of C. G. Park and D. Y. Shin, respectively. Approximate higher derivations and approximate generalized higher derivations on a multi-Banach algebras were investigated by Moslehian [53] and Shateri [55], respectively; see also [68, 69] for some stability results concerning the approximate derivations of order $n$. Moreover, Fošner established the generalized HyersUlam-Rassias stability of (generalized) module left $(m, n)$ derivations on a normed algebra $\mathscr{A}$ into a Banach left $\mathscr{A}$ module $[50,51]$ and the stability of $(m, n)_{(\delta, \tau)}$-derivations on a normed algebra $\mathscr{A}$ into a Banach $\mathscr{A}$-bimodule [66] (see also [70] for a classical result in this regard).

For the stability results of ternary derivations, we refer to [52] (see also [30]), where some recent results are discussed. Moreover, using the fixed point method, Moslehian [52] established the generalized Hyers-Ulam-Rassias stability of ternary derivations, from a normed ternary algebra into a Banach trimodule, associated with the generalized Jensen functional equations

$$
r f\left(\frac{s x+t y}{r}\right)=s f(x)+t f(y)
$$

where $r, s, t$ are given constants and $f$ is an unknown function. Let us point out that a mapping $f: X \rightarrow Y$ between linear spaces $\mathscr{X}$ and $\mathscr{Y}$ with $f(0)=0$ satisfies the above generalized Jensen equation if and only if it is additive (cf. [71]).

Finally, let us mention that some other results, which have not been discussed in this paper (but somehow concern stability of derivations), can be found in [31, 72-76].

\section{Competing Interests}

The authors declare that they have no competing interests.

\section{References}

[1] S. M. Ulam, A Collection of Mathematical Problems, Interscience Publishers, New York, NY, USA, 1960.

[2] S. M. Ulam, Problems in Modern Mathematics, John Wiley \& Sons, New York, NY, USA, 1964.
[3] D. H. Hyers, "On the stability of the linear functional equation," Proceedings of the National Academy of Sciences of the United States of America, vol. 27, pp. 222-224, 1941.

[4] T. Aoki, "On the stability of the linear transformation in Banach spaces," Journal of the Mathematical Society of Japan, vol. 2, pp. 64-66, 1950.

[5] T. M. Rassias, "On the stability of the linear mapping in Banach spaces," Proceedings of the American Mathematical Society, vol. 72, no. 2, pp. 297-300, 1978.

[6] J. Brzdęk, W. Fechner, M. S. Moslehian, and J. Sikorska, "Recent developments of the conditional stability of the homomorphism equation," Banach Journal of Mathematical Analysis, vol. 9, no. 3, pp. 278-326, 2015.

[7] D. H. Hyers, G. Isac, and T. M. Rassias, Stability of Functional Equations in Several Variables, vol. 34 of Progress in Nonlinear Differential Equations and their Applications, Birkhäuser Boston, Boston, Mass, USA, 1998.

[8] S.-M. Jung, Hyers-Ulam-Rassias Stability of Functional Equations in Nonlinear Analysis, vol. 48 of Springer Optimization and Its Applications, Springer, New York, NY, USA, 2011.

[9] T. M. Rassias, Ed., Functional Equations, Inequalities and Applications, Kluwer Academic, Dordrecht, The Netherlands, 2003.

[10] M. Brešar, "On the distance of the composition of two derivations to the generalized derivations," Glasgow Mathematical Journal, vol. 33, no. 1, pp. 89-93, 1991.

[11] M. Mathieu, Ed., Elementary Operators \& Applications. In Memory of Domingo A. Herrero. Proceedings of the International Workshop held in Blaubeuren, June 912, 1991, World Scientific, River Edge, NJ, USA, 1992.

[12] B. Hvala, "Generalized derivations in rings," Communications in Algebra, vol. 26, no. 4, pp. 1147-1166, 1998.

[13] J. Vukman, "A note on generalized derivations of semiprime rings," Taiwanese Journal of Mathematics, vol. 11, no. 2, pp. 367370, 2007.

[14] I. M. Singer and J. Wermer, "Derivations on commutative normed algebras," Mathematische Annalen, vol. 129, pp. 260 264, 1955.

[15] M. P. Thomas, "The image of a derivation is contained in the radical," Annals of Mathematics, vol. 128, no. 3, pp. 435-460, 1988.

[16] B. E. Johnson, "Continuity of derivations on commutative algebras," American Journal of Mathematics, vol. 91, pp. 1-10, 1969.

[17] O. Hatori and J. Wada, "Ring derivations on semi-simple commutative Banach algebras," Tokyo Journal of Mathematics, vol. 15, no. 1, pp. 223-229, 1992.

[18] T. Miura, G. Hirasawa, and S.-E. Takahasi, "A perturbation of ring derivations on Banach algebras," Journal of Mathematical Analysis and Applications, vol. 319, no. 2, pp. 522-530, 2006.

[19] P. Šemrl, "The functional equation of multiplicative derivation is superstable on standard operator algebras," Integral Equations and Operator Theory, vol. 18, no. 1, pp. 118-122, 1994.

[20] K.-W. Jun and D.-W. Park, "Almost derivations on the Banach algebra $C^{n}[0,1]$, , Bulletin of the Korean Mathematical Society, vol. 33, no. 3, pp. 359-366, 1996.

[21] B. E. Johnson, "Approximately multiplicative functionals," Journal of the London Mathematical Society. Second Series, vol. 34, no. 3, pp. 489-510, 1986.

[22] B. E. Johnson, "Approximately multiplicative maps between Banach algebras," Journal of the London Mathematical Society. Second Series, vol. 37, no. 2, pp. 294-316, 1988. 
[23] K. Jarosz, Perturbations of Banach Algebras, Springer, Berlin, Germany, 1985.

[24] K. Jarosz, "Ultraproducts and small bound perturbations," Pacific Journal of Mathematics, vol. 148, no. 1, pp. 81-88, 1991.

[25] M. Amyari and M. S. Moslehian, "Hyers-Ulam-Rassias stability of derivations on Hilbert $C^{*}$-modules," in Topological Algebras and Applications, vol. 427 of Contemporary Mathematics, pp. 3139, American Mathematical Society, Providence, RI, USA, 2007.

[26] R. Badora, "On approximate derivations," Mathematical Inequalities \& Applications, vol. 9, no. 1, pp. 167-173, 2006.

[27] M. E. Gordji and M. S. Moslehian, "A trick for investigation of approximate derivations," Mathematical Communications, vol. 15, no. 1, pp. 99-105, 2010.

[28] C.-G. Park, "Linear derivations on Banach algebras," Nonlinear Functional Analysis and Applications, vol. 9, no. 3, pp. 359-368, 2004.

[29] C.-G. Park, "Lie *-homomorphisms between Lie $C^{*}$-algebras and Lie $*$-derivations on Lie $C^{*}$-algebras," Journal of Mathematical Analysis and Applications, vol. 293, no. 2, pp. 419-434, 2004.

[30] M. Amyari, C. Baak, and M. S. Moslehian, "Nearly ternary derivations," Taiwanese Journal of Mathematics, vol. 11, no. 5, pp. 1417-1424, 2007.

[31] F. Polat, "Approximate Riesz algebra-valued derivations," Abstract and Applied Analysis, vol. 2012, Article ID 240258, 5 pages, 2012.

[32] G. Isac and T. M. Rassias, "On the Hyers-Ulam stability of $\psi$ additive mappings," Journal of Approximation Theory, vol. 72, no. 2, pp. 131-137, 1993.

[33] Z. Gajda, “On stability of additive mappings," International Journal of Mathematics and Mathematical Sciences, vol. 14, no. 3, pp. 431-434, 1991.

[34] M. S. Moslehian, "Hyers-Ulam-Rassias stability of generalized derivations," International Journal of Mathematics and Mathematical Sciences, vol. 2006, Article ID 93942, 8 pages, 2006.

[35] R. Badora, "On approximate ring homomorphisms," Journal of Mathematical Analysis and Applications, vol. 276, no. 2, pp. 589597, 2002.

[36] H. G. Dales, Banach Algebras and Automatic Continuity, The Clarendon Press, Oxford University Press, New York, NY, USA, 2000.

[37] M. E. Gordji and M. Filali, "Arens regularity of module actions," Studia Mathematica, vol. 181, no. 3, pp. 237-254, 2007.

[38] A. Fošner and M. S. Moslehian, "On approximate generalized derivations," in The Natália Bebiano Anniversary Volume, vol. 44 of Textos de Matematica Série B, pp. 33-44, University of Coimbra, Coimbra, Portugal, 2013.

[39] J. A. Baker, "The stability of the cosine equation," Proceedings of the American Mathematical Society, vol. 80, no. 3, pp. 411-416, 1980.

[40] S.-M. Jung, M. T. Rassias, and C. Mortici, "On a functional equation of trigonometric type," Applied Mathematics and Computation, vol. 252, pp. 294-303, 2015.

[41] Z. Moszner, "Sur les définitions différentes de la stabilité des équations fonctionnelles," Aequationes Mathematicae, vol. 68, no. 3, pp. 260-274, 2004.

[42] D. G. Bourgin, "Approximately isometric and multiplicative transformations on continuous function rings," Duke Mathematical Journal, vol. 16, pp. 385-397, 1949.

[43] Gy. Maksa and Zs. Páles, "Hyperstability of a class of linear functional equations," Acta Mathematica Academiae Paedagogiace Nyíregyháziensis, New Series, vol. 17, no. 2, pp. 107-112, 2001.
[44] E. Gselmann, "Hyperstability of a functional equation," Acta Mathematica Hungarica, vol. 124, no. 1-2, pp. 179-188, 2009.

[45] Gy. Maksa, K. Nikodem, and Zs. Páles, "Results on $t$-Wright convexity," Comptes Rendus Mathématiques des l'Académie des Sciences. La Société Royale du Canada, vol. 13, no. 6, pp. 274278, 1991.

[46] N. Brillouët-Belluot, J. Brzdęk, and K. Ciepliński, "On some recent developments in Ulam's type stability," Abstract and Applied Analysis, vol. 2012, Article ID 716936, 41 pages, 2012.

[47] J. Brzdęk and K. Ciepliński, "Hyperstability and superstability," Abstract and Applied Analysis, vol. 2013, Article ID 401756, 13 pages, 2013.

[48] H.-X. Cao, J.-R. Lv, and J. M. Rassias, "Superstability for generalized module left derivations and generalized module derivations on a Banach module (I)," Journal of Inequalities and Applications, vol. 2009, Article ID 718020, 10 pages, 2009.

[49] H.-X. Cao, J.-R. Lv, and J. M. Rassias, "Superstability for generalized module left derivations and generalized module derivations on a Banach module. II," Journal of Inequalities in Pure and Applied Mathematics, vol. 10, no. 3, article 85, 8 pages, 2009.

[50] A. Fošner, "On the generalized Hyers-Ulam stability of module left $(m, n)$-derivations," Aequationes Mathematicae, vol. 84, no. 1-2, pp. 91-98, 2012.

[51] A. Fošner, "Hyers-Ulam-Rassias stability of generalized module left $(m, n)$-derivations," Journal of Inequalities and Applications, vol. 2013, article 2013:208, 8 pages, 2013.

[52] M. S. Moslehian, "Ternary derivations, stability and physical aspects," Acta Applicandae Mathematicae, vol. 100, no. 2, pp. 187-199, 2008.

[53] M. S. Moslehian, "Superstability of higher derivations in multiBanach algebras," Tamsui Oxford Journal of Mathematical Sciences, vol. 24, no. 4, pp. 417-427, 2008.

[54] C.-G. Park, M. E. Gordji, and Y. J. Cho, "Stability and superstability of generalized quadratic ternary derivations on nonArchimedean ternary Banach algebras: a fixed point approach," Fixed Point Theory and Applications, vol. 2012, article 2012:97, 8 pages, 2012.

[55] T. L. Shateri, "Superstability of generalized higher derivations," Abstract and Applied Analysis, vol. 2011, Article ID 239849, 9 pages, 2011.

[56] M. Kuczma, An Introduction to the Theory of Functional Equations and Inequalities. Cauchy's Equation and Jensen's Inequality, Birkhäuser, Basel, Switzerland, 2009.

[57] W. Jabłoński, "On a class of sets connected with a convex function," Abhandlungen aus dem Mathematischen Seminar der Universität Hamburg, vol. 69, pp. 205-210, 1999.

[58] W. Jabłoński, "Sum of graphs of continuous functions and boundedness of additive operators," Journal of Mathematical Analysis and Applications, vol. 312, no. 2, pp. 527-534, 2005.

[59] T. Miura, H. Oka, G. Hirasawa, and S.-E. Takahasi, "Superstability of multipliers and ring derivations on Banach algebras," Banach Journal of Mathematical Analysis, vol. 1, no. 1, pp. 125130, 2007.

[60] A. Najati and C. Park, "Stability of homomorphisms and generalized derivations on Banach algebras," Journal of Inequalities and Applications, vol. 2009, Article ID 595439, 12 pages, 2009.

[61] J. Brzdęk and A. Fošner, "On approximate generalized Lie derivations," Glasnik Matematicki. Serija III, vol. 50, no. 1, pp. 77-99, 2015. 
[62] M. Ashraf and W. Al-Shammakh, "On generalized $(\theta, \phi)$ derivations in rings," International Journal of Mathematics, Game Theory and Algebra, vol. 12, no. 4, pp. 295-300, 2002.

[63] M. Mirzavaziri and M. S. Moslehian, "Automatic continuity of $\sigma$-derivations on $C^{*}$-algebras," Proceedings of the American Mathematical Society, vol. 134, no. 11, pp. 3319-3327, 2006.

[64] M. Amyari, F. Rahbarnia, and G. Sadeghi, "Some results on stability of extended derivations," Journal of Mathematical Analysis and Applications, vol. 329, no. 2, pp. 753-758, 2007.

[65] C. Baak and M. S. Moslehian, "On the stability of $\theta$-derivations on $J B^{*}$-triples," Bulletin of the Brazilian Mathematical Society, vol. 38, no. 1, pp. 115-127, 2007.

[66] A. Fošner, "The Hyers-Ulam-Rassias stability of $(m, n)_{(\sigma, \tau)}$ derivations on normed algebras," Abstract and Applied Analysis, vol. 2012, Article ID 347478, 11 pages, 2012.

[67] M. S. Moslehian, "Approximate $(\sigma, \tau)$-contractibility," Nonlinear Functional Analysis and Applications, vol. 11, no. 5, pp. 805-813, 2006.

[68] J. Schwaiger, "On the stability of derivations of higher order," Annales Academiae Paedagogicae Cracoviensis Studia Mathematica, vol. 4, pp. 139-146, 2001.

[69] E. Gselmann, "Approximate derivations of order n," Acta Mathematica Hungarica, vol. 144, no. 1, pp. 217-226, 2014.

[70] Y.-S. Jung, "On the generalized Hyers-Ulam stability of module left derivations," Journal of Mathematical Analysis and Applications, vol. 339, no. 1, pp. 108-114, 2008.

[71] D.-H. Boo, S.-Q. Oh, C.-G. Park, and J.-M. Park, "Generalized Jensen's equations in Banach modules over a $C^{*}$-algebra and its unitary group," Taiwanese Journal of Mathematics, vol. 7, no. 4, pp. 641-655, 2003.

[72] K.-W. Jun and H.-M. Kim, "Approximate derivations mapping into the radicals of Banach algebras," Taiwanese Journal of Mathematics, vol. 11, no. 1, pp. 277-288, 2007.

[73] C. Park and D.-H. Boo, "Isomorphisms and generalized derivations in proper CQ* -algebras," Journal of Nonlinear Science and Its Applications, vol. 4, no. 1, pp. 19-36, 2011.

[74] C. Park, D.-H. Boo, and J. S. An, "Homomorphisms between $C^{*}$-algebras and linear derivations on $C^{*}$-algebras," Journal of Mathematical Analysis and Applications, vol. 337, no. 2, pp. 14151424, 2008.

[75] J. Roh and I.-S. Chang, "Approximate derivations with the radical ranges of noncommutative Banach algebras," Abstract and Applied Analysis, vol. 2015, Article ID 594075, 7 pages, 2015.

[76] T. L. Shateri and G. Sadeghi, "Stability of derivations in modular spaces," Mediterranean Journal of Mathematics, vol. 11, no. 3, pp. 929-938, 2014. 


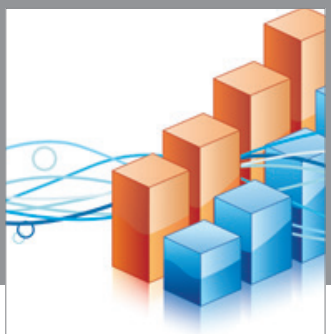

Advances in

Operations Research

vatem alat4

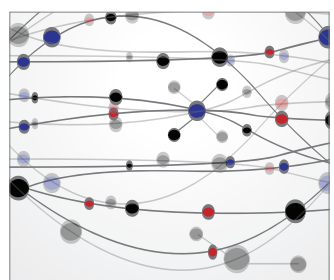

\section{The Scientific} World Journal
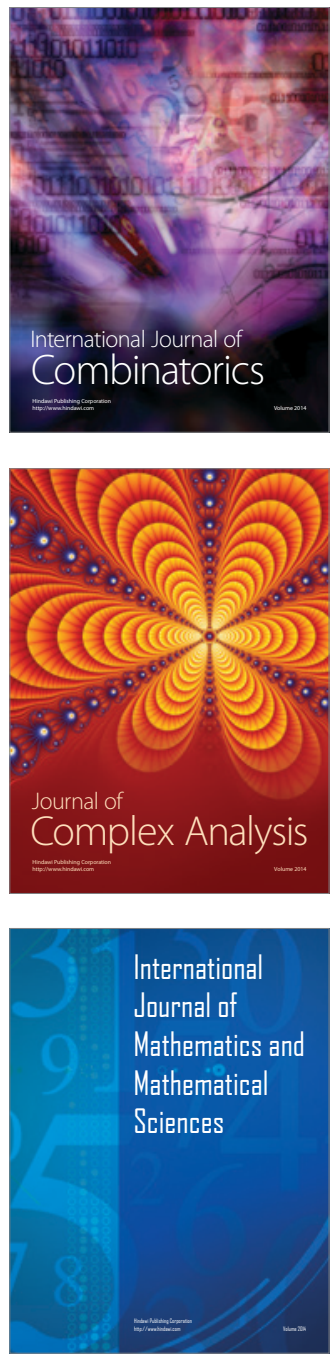
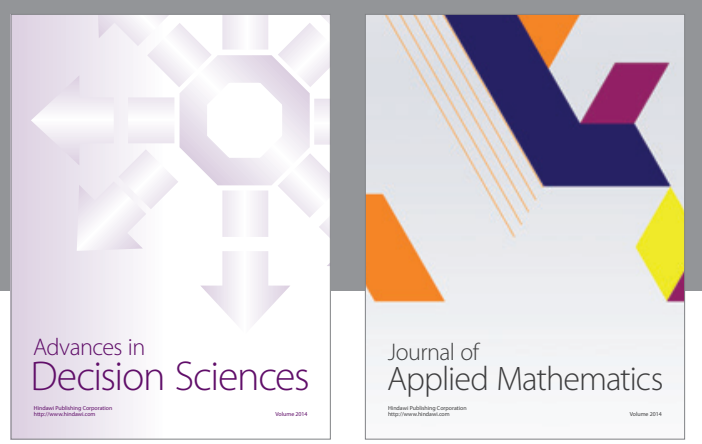

Algebra

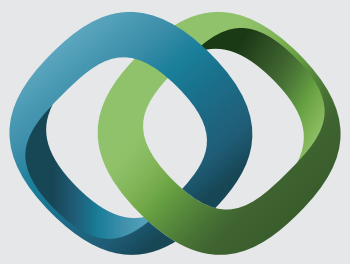

\section{Hindawi}

Submit your manuscripts at

http://www.hindawi.com
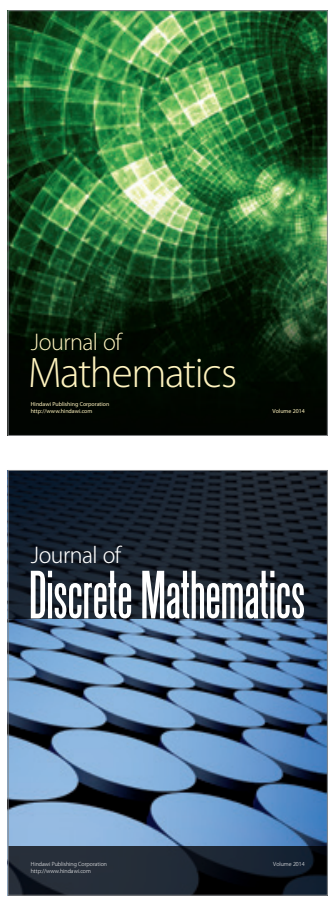

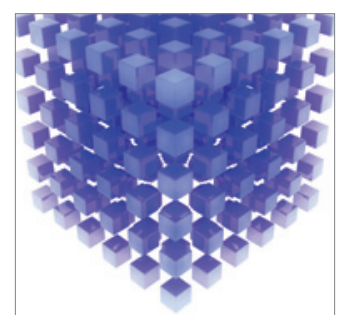

Mathematical Problems in Engineering
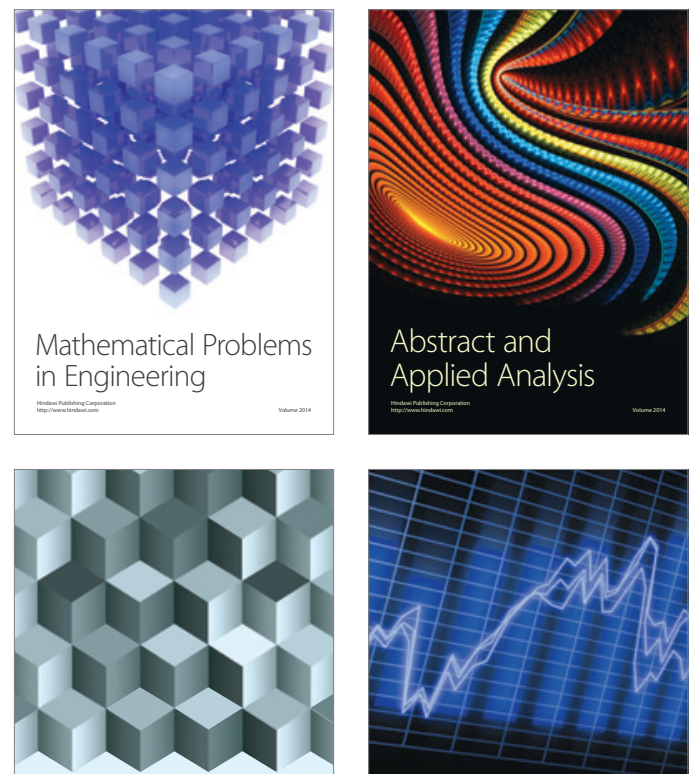

Journal of

Function Spaces

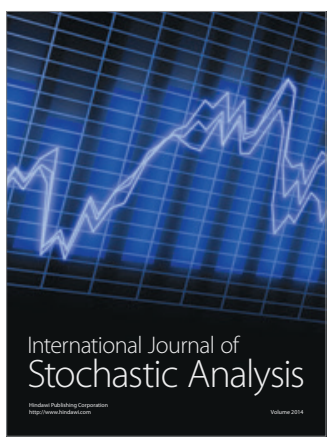

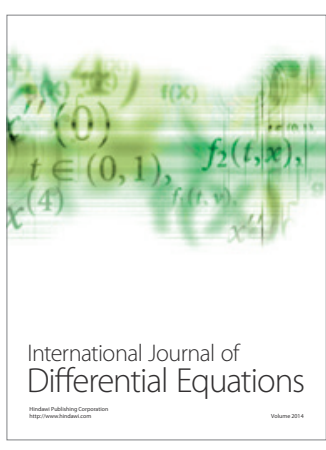
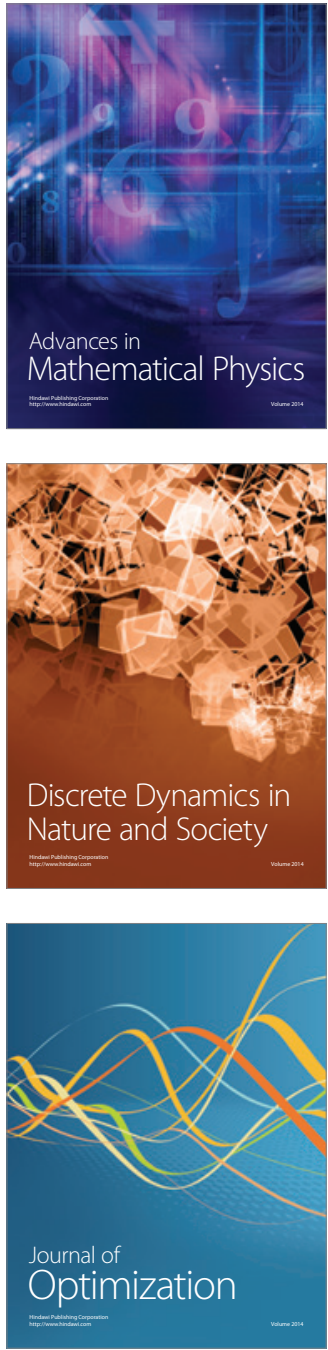